\section{Response to: 'Revised 2017 international consensus on ANCA testing in small vessel vasculitis: support from an external quality assessment' by Broeders et al}

The correspondence by Broeders et $a l^{1}$ is very welcome because it is seeking further support for the revised 2017 international consensus on ANCA testing in small vessel vasculitis. $^{2}$ The support is based on the results of an external quality assessment (EQA) in Belgium. Based on a single sample from a patient strongly suspected of having antineutrophil cytoplasmic antibodies (ANCA)-associated vasculitis (AAV), it was concluded that the real-life performance of antigen-specific immunoassays is better than the performance of indirect immunofluorescence (IIF) testing. Besides this, interesting information about the testing algorithm and type of immunoassay in the Belgian clinical laboratories is also provided. The main question is, however, whether solid conclusions can be drawn from the results obtained by the EQA.

Among the 90 participating laboratories, 13 laboratories only use antigen-specific immunoassays and apparently have abandoned IIF, as advocated in the revised 2017 consensus. Seventy laboratories still use the combination of IIF and immunoassays according to the 1999 international consensus statement on ANCA testing, ${ }^{4}$ while seven laboratories only perform IIF and therefore seem not to have accepted neither the 1999 consensus nor the revised 2017 consensus. It is questionable, however, if such deductions with respect to compliance to consensus statements can be drawn from the EQA rounds. First, although it is tempting to speculate that the 13 laboratories (17\%) that only perform antigen-specific immunoassays already have adopted the revised 2017 consensus, a previous inventory on ANCA algorithms in European laboratories revealed that $14 \%$ of the participating Belgian laboratories $(n=72)$ did not perform ANCA IIF testing. ${ }^{5}$ Since this questionnaire-based study was performed a few years before the revised 2017 consensus became available, implementation of the new consensus in Belgian laboratories may still be limited. Second, laboratories that used both IIF and antigen-specific immunoassays in the respective EQA round may currently use only immunoassays in daily clinical practice for the diagnostic work-up of AAV. Since ANCA IIF may be of added value for gastrointestinal autoimmune diseases, IIF might have been performed on the EQA sample because participation in the EQA for each clinical laboratory parameter is obligatory for ISO 15189 accreditation. Third, laboratories that only perform IIF may use this as a screening assay for selecting samples to be further tested by antigen-specific immunoassays in a referral laboratory, and as such still follow the 1999 consensus.

Although ANCA testing algorithms used for the EQA rounds may deviate from daily clinical practice, as suggested above, the situation described by Broeders et al is almost identical to the data obtained in the questionnaire-based study. ${ }^{15}$ About $85 \%$ of the Belgian laboratories perform ANCA IIF tests, and this is probably also driven by the national reimbursement policy that requires IIF testing prior to antigen-specific immunoassays. As such, accommodation to the revised 2017 consensus requires adjustment of these rules, at least in Belgium, but likely also in other countries.
Besides the data about the ANCA testing algorithm, the types of immunoassay used for the EQA are also very similar to the situation described in the questionnaire-based study. ${ }^{15}$ About 20\% of the Belgian laboratories use dot or line immunoassays. Although some of these assays may reveal categorical semiquantitative data based on scanning results, it will be more difficult to interpret results in terms of likelihood ratios for test result intervals, as advocated in the revised 2017 consensus. $^{36}$

Finally, and probably most importantly, it should be recognised that the EQA is not intended to evaluate the clinical performance of the tests included. The main goal of the EQA is to establish if the participating laboratories use state-of-the-art procedures. Samples included are often hampered by a selection bias: most samples are chosen because unequivocal results are expected. If discrepant results are obtained for such samples, this hints at inadequate laboratory procedures. Incidentally, a sample is chosen because it is known in advance that discrepant results will be obtained. Such samples may give information about the performance of distinct assays for the respective samples, but further extrapolation of the data is not valid. In case of the eLetter of Broeders et al, ${ }^{1}$ only a single sample with MPO-ANCA is evaluated. Since all EQA participants that use antigen-specific immunoassays report MPO-ANCA, it is very likely that the respective laboratories adequately follow their procedures. However, this does not imply that the distinct immunoassays used share similar test characteristics. For this to conclude, a large series of samples is required that is representative of the large variety of clinical manifestations observed in $\mathrm{AAV}^{2}$ Similarly, although the respective MPO-ANCA sample seems to support the revised 2017 consensus, it would not have been too difficult to select a sample that only is positive by IIF. Evidently, this should not be accepted as an argument to refute the revised 2017 consensus.

Altogether, it is very important that the revised 2017 international consensus on ANCA testing in small vessel vasculitis will be validated, preferentially in a prospective multicentre study. As argued in this response to the eLetter of Broeders et al, ${ }^{1}$ data obtained by the EQA have serious limitations with respect to conclusions about assay performance and testing algorithms.

\section{Jan Damoiseaux}

Central Diagnostic Laboratory, Maastricht University Medical Center, Maastricht, The Netherlands

Correspondence to Dr Jan Damoiseaux, Central Diagnostic Laboratory, Maastricht University Medical Center, Maastricht 6229 HX, The Netherlands; jan.damoiseaux@mumc.nl

Handling editor Professor Josef S Smolen

Contributors This is a single-author response and I prepared the manuscript myself.

Funding The authors have not declared a specific grant for this research from any funding agency in the public, commercial or not-for-profit sectors.

Competing interests None declared.

Patient consent Not required.

Provenance and peer review Commissioned; internally peer reviewed.

(c) Author(s) (or their employer(s)) 2019. No commercial re-use. See rights and permissions. Published by BMJ.

Check for updates

To cite Damoiseaux J. Ann Rheum Dis 2019;78:e114. 
Received 13 August 2018

Accepted 13 August 2018

Published Online First 5 September 2018

\section{(5) Linked}

- http://dx.doi.org/10.1136annrheumdis-2018-214078

Ann Rheum Dis 2019;78:e114. doi:10.1136/annrheumdis-2018-214243

\section{REFERENCES}

1 Broeders S, Goletti S, Tomasi I-P. The revised 2017 international consensus on ANCA testing in small vessel vasculitis: support from an external quality assessment. Ann Rheum Dis 2019;78:e113-e113.
2 Damoiseaux J, Csernok E, Rasmussen N, et al. Detection of antineutrophil cytoplasmic antibodies (ANCAs): a multicentre European Vasculitis Study Group (EUVAS) evaluation of the value of indirect immunofluorescence (IIF) versus antigen-specific immunoassays. Ann Rheum Dis 2017:76:647-53.

3 Bossuyt X, Cohen Tervaert JW, Arimura Y, et al. Position paper: revised 2017 international consensus on testing of ANCAs in granulomatosis with polyangiitis and microscopic polyangiitis. Nat Rev Rheumatol 2017;13:683-92.

4 Savige J, Gillis D, Benson E, et al. International consensus statement on testing and reporting of Antineutrophil Cytoplasmic Antibodies (ANCA). Am J Clin Pathol 1999;111:507-13.

5 Damoiseaux J, Heijnen I, Van Campenhout C, et al. An international survey on antineutrophil cytoplasmic antibodies (ANCA) testing in daily clinical practice. Clin Chem Lab Med 2017.

6 Bossuyt X, Rasmussen N, van Paassen P, et al. A multicentre study to improve clinical interpretation of proteinase-3 and myeloperoxidase anti-neutrophil cytoplasmic antibodies. Rheumatology 2017;56:1533-41. 